\title{
O princípio do direito humano à alimentação adequada e a doença celíaca: avanços e desafios
}

\section{The principle of human right to adequate food and celiac disease: advancements and challenges}

Juliana Nadal'

Sila Mary Rodrigues Ferreira ${ }^{2}$

Islandia Bezerra Da Costa²

Suely Teresinha Schmidt²

'Departamento de Nutrição. Área Qualidade dos
Alimentos e Nutrição. Universidade Federal do
Paraná. Curitiba, PR, Brasil
${ }^{2}$ Programa de Pós-Graduação em Segurança
Alimentar e Nutricional. Universidade Federal do
Paraná, Curitiba, PR, Brasil
Correspondência / Correspondence
Juliana Nadal
E-mail: junadal20@gmail.com

\section{Resumo}

A doença celíaca se caracteriza pela atrofia parcial ou total das vilosidades intestinais, causada pela ingestão de glúten, presente no trigo, centeio, cevada, aveia e malte. Possui prevalência média de $1 \%$ na população geral. A terapia nutricional recomendada é a retirada completa do glúten da dieta, o que faz com que os sintomas regridam e o estado nutricional seja restabelecido. Este artigo busca contextualizar a insegurança alimentar e nutricional a que estão submetidos os indivíduos com doença celíaca, mais especificamente no que diz respeito ao princípio do direito humano à alimentação adequada (DHAA). Este, por sua vez, estabelece como um direito humano fundamental a disponibilidade de alimentos em quantidade e qualidade e, ainda, que preze pelo bem-estar promovendo a saúde de toda a população. No entanto, a pouca disponibilidade de produtos destinados aos celíacos no mercado, o custo elevado e a contaminação destes por glúten contribuem para uma situação constante de insegurança alimentar e nutricional aos portadores desta necessidade alimentar especial. A dieta é a única e a mais segura forma de tratamento para esta doença, de modo a possibilitar seu controle. Pode-se dizer, portanto, que são necessárias ações, ou ainda, políticas públicas que materializem este direito, garantindo assim acesso a uma alimentação adequada a essa parcela da população.

Palavras-chave: Doença Celíaca. Segurança Alimentar e Nutricional. Dieta Livre de Glúten. 


\section{Abstract}

Celiac disease is characterized by partial or total intestinal villous atrophy, caused by gluten ingestion in wheat, rye, barley, malt and oats. In the general population, the prevalence average is about $1 \%$. The recommended nutritional therapy is the complete removal of gluten from the diet, since this causes the symptoms regress and nutritional status is restored. This paper seeks to contextualize the food and nutrition insecurity that afflicts individuals with celiac disease, specifically with regard to the principle of the Human Right to Adequate Food (HRAF). This, in turn, establishes as a fundamental human right to food availability in quantity and quality, and also values welfare, promoting the health of the entire population. However, the limited availability of products intended for celiac individuals in the market, the high cost and the gluten contamination, contribute to a constant situation of food and nutritional insecurity to holders of this special dietary need. Diet is the single most secure treatment form for this disease, enabling its control. It can be said, therefore, that the public needs actions, or even public policies to materialize this right, thus ensuring access to adequate food to this population.

Key words: Celiac Disease. Food Security. Diet. Gluten-Free.

\section{Introdução}

A doença celíaca (DC) pode ser considerada a intolerância alimentar mais comum do mundo. Em um breve histórico, Aaron ${ }^{1}$ explicita que se trata de uma condição autoimune que acompanha o indivíduo ao longo de toda sua vida. Depois do diagnóstico, o indivíduo celíaco passa a conviver com uma dieta restritiva, já que a maioria dos produtos possui o trigo como principal constituinte e este, por sua vez, tem na sua composição o glúten. O glúten é a proteína de armazenamento do trigo e suas gliadinas, solúveis em álcool, são responsáveis pela instalação da doença. O centeio, a cevada, o malte e a aveia possuem moléculas estruturalmente relacionadas às da gliadina do trigo e, desse modo, a ingestão de glúten desencadeia a doença, pois há resistência da digestão do mesmo pelas enzimas digestivas, desenvolvendo o processo imune ${ }^{2}$ que compromete o trato gastrintestinal, em especial o intestino delgado, de pessoas geneticamente predispostas. ${ }^{1}$

Araújo \& Araújo, ${ }^{3}$ por sua vez, afirmam que a dieta do celíaco deve ser completamente livre de glúten, já que a orientação dietética é o que lhe permite ter uma vida relativamente sem maiores 
complicações patológicas. Para tanto, reforçam a necessidade de os familiares assumirem, também para si, as mesmas regras em sua alimentação cotidiana. No entanto, é preciso ter clareza de que a dieta possui caráter complexo e restritivo. Tem um custo elevado, quando comparado ao que se considera uma dieta convencional, ${ }^{4}$ o que leva o celíaco a ter dificuldade na disponibilidade e no acesso ao alimento sem glúten. Esta condição infringe o princípio do direito humano à alimentação adequada, pois condiciona ao indivíduo celíaco a uma situação permanente de insegurança alimentar e nutricional, o que pode trazer prejuízo na qualidade de vida, na socialização e na saúde do indivíduo, tanto em curto como no longo prazo.

Neste sentido, o presente artigo tem como objetivo contextualizar e problematizar a situação de insegurança alimentar e nutricional a que estão submetidos os indivíduos com doença celíaca, mais especificamente no que diz respeito ao princípio do direito humano à alimentação adequada (DHAA) sob a ótica da Segurança Alimentar e Nutricional (SAN) .

\section{Métodos}

Foi realizada pesquisa bibliográfica nas bases de dados Science Direct e SciELO, utilizando-se os seguintes descritores, em português e inglês: doença celíaca, segurança alimentar e nutricional, dieta sem glúten, epidemiologia, tratamento e direito humano à alimentação adequada. Também foram analisadas a legislação vigente e outros documentos oficiais sobre Segurança Alimentar e Nutricional. Não houve limite de data para as pesquisas.

Os critérios utilizados para seleção dos artigos foram a abordagem da doença celíaca, a segurança alimentar e nutricional e o direito humano à alimentação adequada, sendo excluídos da pesquisa os materiais bibliográficos que não abordavam diretamente o escopo desta revisão, embora apresentassem os descritores selecionados.

O universo do estudo foi constituído por 97 artigos, dos quais 48 foram excluídos em uma primeira triagem realizada por meio da leitura dos resumos, e 17 depois da leitura completa. Para a elaboração do presente artigo, foram utilizadas 32 referências.

\section{Doença celíaca: epidemiologia e manifestações clínicas}

Alguns dados epidemiológicos referem que na Europa e nos Estados Unidos, a prevalência de DC encontra-se entre 0,5 e 1,0\%. ${ }^{5}$ Esta mesma prevalência ocorre no oeste indiano e nas populações árabes. ${ }^{6}$ Embora esteja presente em uma a cada 100 pessoas, estima-se que apenas 10 a $15 \%$ dos celíacos são devidamente diagnosticados. ${ }^{7}$ 
Contudo, ressalta-se que a prevalência de casos dessa doença depende da região em estudo e também da metodologia empregada. Estudos realizados em doadores de sangue mostraram que nos Estados Unidos, por exemplo, a frequência de casos é de 1:250; já no Brasil, no Distrito Federal, é de 1:681, e em Ribeirão Preto, 1:273. ${ }^{8}$ A DC ocorre predominantemente em indivíduos caucasianos, sendo que os não caucasianos representam uma parcela mínima da população com essa enfermidade. ${ }^{8}$ Familiares de primeiro grau de portadores de DC são os indivíduos com maior chance de desenvolver a doença, com frequência de 10 a 20 vezes maior que a população em geral, pois compartilham fatores de risco ambientais e genéticos para a DC. ${ }^{9}$

As formas clínicas da DC vêm sendo comparadas a um iceberg, no qual a forma clássica da doença fica na ponta, enquanto que as formas não clássicas ou assintomáticas permanecem submersas. ${ }^{10}$ Assim, somente pacientes com a forma clássica da doença são diagnosticados, enquanto que os demais permanecem sem diagnóstico. Este fato acaba não gerando as notificações necessárias para uma maior e melhor compreensão e atuação, por parte do Estado e da sociedade, em prol de maiores investimentos no que diz respeito, sobretudo, a estratégias de elaboração e disponibilidade de produtos alimentícios que garantam a inocuidade necessária para os indivíduos com DC.

Para Strauch \& Cotter, ${ }^{11}$ a doença celíaca clássica possui sintomas gastrointestinais variáveis, sendo os mais comuns a diminuição do peso corporal sem motivo, falta de apetite, diarreia, constipação, esteatorreia, vômitos, inchaço e dores abdominais. No entanto, os autores afirmam também que grande parte do diagnóstico realizado em adultos é relativa à forma não clássica da doença ou assintomática. Em geral, os primeiros sinais e sintomas relacionados às formas não clássica e assintomática da doença envolvem manifestações endócrinas, hematológicas, neurológicas, psiquiátricas, reumatológicas e dermatológicas, não incluindo as do trato gastrointestinal. Já Kumar et al. ${ }^{6}$ trazem a constatação de que a forma clássica da doença com os sintomas como diarreia e má absorção são menos comuns, enquanto que as formas não clássica e assintomática têm aumentado. Esta maior prevalência se explica pelo fato de que o diagnóstico (testes sorológicos) para esta doença se tornou mais acessível e houve maior conscientização na prática clínica.

A ingestão de glúten por pessoas com DC pode trazer inúmeros males à saúde, como é o caso da má absorção de nutrientes que são essenciais para a manutenção fisiológica do organismo - neste caso podem-se citar o ferro, o ácido fólico, o cálcio e as vitaminas lipossolúveis. Também pode ser considerada como fator de risco para o desencadeamento de outras doenças crônicas como, por exemplo, diabetes tipo 1, doença autoimune da tireoide, artrite reumatoide, doença de Addison, síndrome de Sjögren, câncer intestinal, osteoporose, infertilidade em mulheres, enfermidades neurológicas, bem como distúrbios psiquiátricos ${ }^{5}$ e orte. $^{12}$

Ludvigsson et al. ${ }^{13}$ demonstram que existe relação direta da DC com a depressão, sendo que o mecanismo dessa associação pode incluir condições do sistema nervoso central (SNC), comorbidades 
somáticas, baixa qualidade de vida e também dificuldades financeiras devidas a maiores cuidados com a saúde; além disso, há necessidade de uma alimentação "especial”, ou ainda, a combinação desses fatores. Devido à associação da DC com distúrbios psiquiátricos, em especial a depressão, há a probabilidade de que existam maiores riscos que podem levar à ocorrência de suicídios nesse grupo.

No entanto, como já evidenciado anteriormente e agora reforçado pelos estudos de Kotze, ${ }^{14}$ a possibilidade de aderir a uma dieta isenta de glúten faz com que sintomas como os defeitos na absorção de micro e macronutrientes, diarreia e edema desapareçam rapidamente. Isto pode levar dias ou semanas, o que leva ao surgimento do apetite e melhora do estado nutricional. A adesão e manutenção da dieta sem glúten por três a cinco anos fazem com que o risco de mortalidade em relação à população geral desapareça. ${ }^{15}$

Corroborando essa assertiva, é importante salientar que não basta simplesmente aderir a uma dieta qualquer; é necessário que esta seja isenta de glúten e possua atributos que a caracterizem como adequada e saudável nos quesitos variedade e disponibilidade em quantidade e qualidade.

\section{A Segurança Alimentar e Nutricional (SAN) e o princípio do direito humano à alimentação adequada (DHAA)}

A Segurança Alimentar e Nutricional (SAN) tem se tornado uma preocupação mundial e assume uma dimensão cada vez mais complexa, dadas as situações que se apresentam (explícita ou implicitamente). Desta forma, a Política Nacional de Alimentação e Nutrição (PNAN) ${ }^{16}$ deve interagir com a Política Nacional de Segurança Alimentar e Nutricional (PNSAN) e outras políticas de desenvolvimento econômico e social, de modo a desempenhar papel importante na estratégia do desenvolvimento das políticas de SAN, principalmente em assuntos relacionados a diagnóstico, vigilância da situação alimentar e nutricional e promoção da alimentação adequada e saudável para indivíduos eutróficos, como também indivíduos com alimentação especial, como os celíacos.

Assim, conhecer e reconhecer a real necessidade das pessoas que vivem de alguma forma sob a ameaça da insegurança alimentar possibilita pensar e sobretudo agir no processo de construção, implementação e aprimoramento das políticas de saúde,${ }^{17}$ de modo a atender às necessidades da população. É imperativo afirmar que tanto o Estado como a sociedade devem promover ações que viabilizem o acesso a uma alimentação adequada, seja em termos de quantidade, seja em termos de qualidade nutricional, de modo a garantir que as necessidades alimentares, biológicas, sociais e culturais sejam alcançadas. A articulação entre o Sistema Único de Saúde (SUS) e o Sistema Nacional de Segurança Alimentar e Nutricional (SISAN) deve investir em políticas públicas de saúde para facilitar o diagnóstico de doenças e implementar as ações de alimentação e nutrição na atenção básica à saúde, no sentido de promover a SAN e, por consequência, diminuir os 
agravos à saúde dos indivíduos, independentemente da condição social, fisiológica e/ou de doença. Também para a implementação de uma PNAN para os indivíduos celíacos, é necessário que surjam investimentos para o desenvolvimento de pesquisas no sentido de mostrar um diagnóstico da condição de insegurança alimentar e nutricional em que os indivíduos celíacos se encontram. ${ }^{16} \mathrm{O}$ Brasil não dispõe de uma programa específico para diagnóstico precoce e acompanhamento da doença celíaca. Enquanto o diagnóstico não for realizado, a alimentação continuará inadequada e irá contribuir para o adoecimento e morte de indivíduos celíacos.

Nesse sentido, para Pinheiro \& Carvalho, ${ }^{18}$ a alimentação e a nutrição devem ser vistas como um direito humano e social do cidadão. Corroborando esses autores, convém fazer referência ao princípio da SAN como sendo inerente a todos os cidadãos: o direito humano à alimentação adequada (DHAA). Ao compreender a alimentação como um direito humano básico, tornase menos complexo compreender o fato de que a ausência de alimentos que contemplem esta necessidade especial, como é o caso dos celíacos, representa um caso concreto de que este grupo de indivíduos está tendo, constantemente, seu direito - à alimentação adequada - violado e, por sua vez, encontra-se em insegurança alimentar e nutricional.

O desenvolvimento integral do cidadão se refere ao conceito de Segurança Alimentar e Nutricional (SAN), como um direito fundamental que deve garantir o acesso da população a uma vida digna e plena. ${ }^{19}$ A Lei ${ }^{\circ} 11.346$, de 15 de setembro de 2006, em seu Art. $3^{\circ}$ estabelece que

[...] a segurança alimentar e nutricional consiste na realização do direito de todos ao acesso regular e permanente a alimentos de qualidade, em quantidade suficiente, sem comprometer o acesso a outras necessidades essenciais, tendo como base práticas alimentares promotoras de saúde que respeitem a diversidade cultural e que sejam ambiental, cultural, econômica e socialmente sustentáveis. ${ }^{20}$

Nesses termos, esclarece-se que a SAN engloba duas dimensões: a alimentar, que trata da produção, da disponibilidade e do acesso aos alimentos; e a nutricional, que determina as relações entre o alimento, o corpo e o homem: o "DHAA é um direito humano de todos e a garantia da Segurança Alimentar e Nutricional para todos é um dever do Estado e responsabilidade da sociedade". ${ }^{21}$ No Brasil, a definição de SAN ganhou destaque quando incorporou no seu conceito os termos que fazem referência a qualidade e quantidade de alimentos e, ainda, sobre a necessidade de estes serem adequados para toda a população, sem fazer distinção por classe social, especial, cultural, e/ou concepções étnicas e religiosas. ${ }^{22}$

Para Pinheiro \& Carvalho, ${ }^{19}$ o princípio do DHAA deve ser garantido pelo Estado, em especial, mediante ações concretas como as políticas públicas, que viabilizem recursos para aquisição, 
produção, seleção e consumo de alimentos focando cada vez mais em atender a esse direito. No entanto, é preciso considerar o fato de que a composição da alimentação (dieta), as necessidades nutricionais específicas e o aproveitamento biológico dos alimentos (de quem os consome) são fatores que refletem diretamente no estado nutricional do indivíduo, conforme defende Monteiro. ${ }^{23}$ Deste modo, é mister considerar que seguir tal princípio é fundamental para a manutenção do bom estado nutricional e, considerando os celíacos, esses componentes se tornam vitais.

Assim como para outras necessidades alimentares especiais e considerando as especificidades da doença celíaca, o princípio do DHAA traz consigo outras exigências como as características que demandam o processo de produção de alimentos, a disponibilidade, a acessibilidade e o consumo. Sobre a primeira exigência listada, que concerne ao processo produtivo, considera-se primordial a necessidade de disponibilizar alimentos/produtos livres de glúten, bem como garantir que sejam livres de contaminações, sobretudo de resíduos de gliadina. Deste modo, esses serão considerados alimentos/produtos seguros e adequados, que poderão ser devidamente aproveitados pelo organismo por meio de sua absorção.

No entanto, como já referenciado anteriormente, não basta que o alimento seja adequado e disponível; é fundamental que seja acessível financeiramente ao indivíduo. Sabe-se que os produtos industrializados e/ou artesanais livres de glúten, em sua grande maioria, possuem preços elevados. Esta condição pode levar à redução da frequência de consumo, o que pode contribuir para uma monotonia alimentar severa. Sobre esta assertiva Burity et al. ${ }^{21}$ reforçam que

[...] a alimentação adequada implica acesso a alimentos saudáveis que tenham como atributos: acessibilidade física e financeira, sabor, variedade, cor, bem como aceitabilidade cultural como, por exemplo, respeito a questões religiosas, étnicas e às peculiaridades dos diversos grupos e indivíduos.

O direito à alimentação especial adequada aos indivíduos celíacos é corroborada por Burity et al., ${ }^{21}$ quando afirmam que esse tipo de alimentação é inerente a um grupo populacional que possui uma condição fisiológica diferenciada e, portanto, se faz preponderante o acesso físico e financeiro aos alimentos livres de glúten. No entanto, isto não é o bastante; existem questões religiosas e de ordem cultural que estão envolvidas nesse contexto, as quais também precisam ser respeitadas. É importante ressaltar que o tratamento dietético é o único existente para a manutenção da saúde e qualidade de vida dos celíacos, por isso a alimentação adequada a sua condição precisa de fato ser mantida ao longo de toda a vida. No entanto, é preciso considerar o fato de que o consumo dos alimentos deve ser feito de maneira consciente, buscando segurança em termos da qualidade no que se refere a produção, higiene e abastecimento, minimizando possibilidades de riscos para a saúde. ${ }^{19}$ 


\section{As violações do DHAA: dificuldades na alimentação do celíaco}

Ao referenciar o simples ato de se alimentar e, alimentar aos outros Poulain \& Proença ${ }^{24}$ afirmam que a alimentação humana pode (e deve) ser vista como um fenômeno com elevado nível de complexidade, pois envolve fatores psicológicos, fisiológicos e socioculturais. Tal assertiva já foi explicitada por Diez Garcia ${ }^{25}$ nos anos de 1990, quando - ao fazer referência ao ato de se alimentar - afirmou que "por possuir papel preponderante na vida das pessoas, a prática alimentar, que vai desde o preparo até o consumo do alimento, inclui conceitos subjetivos, tais como os culturais, religiosos, socioeconômicos e familiares". De fato, como afirmam Lee e Newman, ${ }^{26}$ comer é mais que um ato fisiológico, é também uma forma de socialização, uma necessidade emocional do indivíduo, sendo o alimento o elo entre indivíduo-amigos-familiares, que por sua vez repercute na convivência social. A alimentação, por si só, nos garante mais que a sensação de sobrevivência e exigência biológica por vitaminas, minerais, macro e micronutrientes.

Corroborando tais assertivas, Araújo et al. ${ }^{27}$ afirmam que os hábitos alimentares se ligam diretamente ao sentimento das pessoas, pois estes se vinculam aos valores familiares e culturais. Desse modo, retomando o foco sobre as necessidades alimentares especiais dos celíacos, os autores explicam a causa do sofrimento pessoal quando existe a necessidade de se restringir determinados alimentos, o que pode simbolizar para o indivíduo um rompimento de afeto.

O tratamento da DC exige que hábitos alimentares sejam alterados e que haja adesão a novas práticas alimentares. Em outro estudo, Araújo \& Araújo ${ }^{3}$ retomam o debate e passam a defender que a dieta dos celíacos depende não apenas do próprio indivíduo que apresenta a doença, mas em grande parte da família, sendo importante informar a todos os interessados quais alimentos devem ou não ser ingeridos. Esses cuidados vão desde a compra dos gêneros no mercado, até o preparo dos alimentos. Os autores também notaram que um grande número de indivíduos celíacos tem pouco acesso às lojas de produtos especiais, o que provavelmente pode ser devido ao fato de os produtos sem glúten possuírem alto custo; ou ainda, pela não disponibilidade de estabelecimentos. Esses indivíduos relataram sua insatisfação não apenas com os preços desses produtos, mas também com a disponibilidade dos mesmos nos supermercados, além de da pouca satisfação quanto ao sabor e textura.

A doença celíaca traz, portanto, dificuldades à vida de seus portadores em situações cotidianas como ter/fazer refeições fora de casa, viajar, trabalhar; impõe ainda limitações de convivência na própria família e promove impacto negativo sobre seu bem-estar e na qualidade de vida de todos os membros. Muitas vezes, em eventos sociais, a dieta acaba sendo interrompida devido à quantidade restrita de alimentos permitidos em sua alimentação, conforme afirmaram Lee e Newman. ${ }^{26}$ Singh \& Whelan ${ }^{28}$ também evidenciaram situações semelhantes e avançam nesta problematização, quando afirmam que a disponibilidade limitada e os maiores custos dos produtos alimentícios sem glúten 
desencadeiam grande impacto no campo financeiro e podem trazer consequências na qualidade de vida do celíaco, comprometendo o cumprimento da dieta sem glúten.

\section{A SAN e o DHAA dos indivíduos celíacos e a democratização dessas concepções}

Se considerarmos que a doença celíaca não escolhe classe social, a situação pode ser mais grave em classes sociais de menor poder aquisitivo, uma vez que a alimentação especial para celíacos é preparada com ingredientes que não possuem incentivo fiscal para redução de preço. Isto traz maior impacto no custo da alimentação, uma vez que os ingredientes sem glúten são mais caros. Esta situação pode ser exemplificada com os 45,8 milhões de pessoas atendidas com a transferência de renda do Programa Bolsa Família. ${ }^{29}$ Se aplicado, na população do Programa Bolsa Família, o resultado da pesquisa com os doadores de sangue na cidade de Brasília, onde foi encontrado 0,14\% de celíacos, a proporção estimada de indivíduos celíacos do Programa Bolsa Família seria de 64.120 pessoas. Este valor pode ser ainda subestimado, uma vez que na amostra referida os doadores de sangue eram predominantemente do sexo masculino e a doença celíaca ocorre mais em mulheres. Da mesma forma, não foi considerado o percentual de $0,54 \%$ de crianças celíacas encontrado na pesquisa do mesmo autor. ${ }^{30}$

Mesmo não havendo dados epidemiológicos oficiais sobre a prevalência da doença celíaca no Brasil, as informações acima, juntamente com a realidade do maior custo da alimentação especial para os celíacos, são fatos que configuram a insegurança alimentar e nutricional dos indivíduos celíacos. Não resta dúvida de que tanto os indivíduos celíacos como a alimentação dos mesmos necessitam de atenção especial.

A dieta restritiva em glúten traz uma série de consequências em suas vidas, já que tais produtos (sem glúten) não são encontrados facilmente e, em geral, são vendidos em lojas de produtos naturais e/ou especiais, na maioria das vezes localizadas nos maiores centros. Além disso, por usarem outros ingredientes substitutos do trigo, o custo desses produtos se torna mais elevado, o que pode torna-los inacessíveis a uma parcela significativa dos celíacos.

A exposição do celíaco ao glúten traz prejuízos às vilosidades intestinais, que se atrofiam e reduzem a capacidade absortiva dos nutrientes, vindo a comprometer o estado nutricional e a saúde do indivíduo. Tais dificuldades ferem o princípio do DHAA desse indivíduo. Para garantir seus direitos, são necessárias ações urgentes voltadas para a solução desses problemas e melhor amparo diante de sua situação de vulnerabilidade alimentar e nutricional. A adequação e adesão à dieta precisam ser totais, para que o celíaco mantenha seu estado nutricional em uma condição de eutrofia e qualidade de vida. Existe, portanto, uma necessidade premente de ações e programas específicos para atender às demandas dos indivíduos celíacos, que tenham como objetivo assegurar seu direito humano básico sob a ótica da Segurança Alimentar e Nutricional. 
Uma medida que poderia ser incorporada à política pública voltada para o indivíduo celíaco seria a redução fiscal (ou mesmo a isenção total de impostos), para que o segmento de produção artesanal e/ou industrial de produto sem glúten seja fomentado, garantindo uma produção sistemática e de qualidade, reduzindo seus custos e permitindo a venda de seus produtos a preços mais acessíveis.

O auxílio financeiro aos celíacos também pode ser outro caminho a ser percorrido. A mesma linha de investimento (social e alimentar) diz respeito à oportunidade de formação e qualificação, mediante oferta de cursos para o aprendizado de técnicas de produção de alimentos sem glúten direcionados aos celíacos e seus familiares. Neste sentido, permite-se maior diversificação alimentar e nutricional, respeitando os aspectos regionais, culturais e sociais, além de assegurar maior qualidade de vida aos celíacos.

O programa social da Prefeitura de Curitiba, Armazém da Família, oferece à população com renda de até $R$ \$1.395,00 e indivíduos com necessidades alimentares especiais, gêneros alimentícios a preço em média $30 \%$ menores que os ofertados em supermercados convencionais. Segundo esse programa, o indivíduo celíaco cadastrado, por meio da comprovação médica de sua necessidade, pode adquirir produtos sem glúten por preços acessíveis.

A adesão do celíaco a uma dieta isenta de glúten é de suma importância para evitar o comprometimento do desenvolvimento pôndero-estrutural e puberal, a fertilidade, a densidade mineral óssea, reduzir as deficiências de micro e macronutrientes e o risco do aparecimento de doenças malignas, em especial do sistema gastrointestinal. No entanto, manter uma dieta totalmente livre de glúten não é tarefa de fácil execução, pois a violação da dieta pode ocorrer de forma voluntária ou involuntária, indo desde a informação incorreta dos rótulos dos alimentos, até a contaminação de produtos industrializados com glúten, como explicitaram Sdepanian et al. ${ }^{31}$

Salienta-se, portanto, que o DHAA deve ser garantido, em especial pelo Estado, mediante políticas públicas que promovam ações e programas visando ao acesso a recursos para aquisição, produção, seleção e consumo de alimentos e focando cada vez mais em atender a este direito, como defendem Pinheiro \& Carvalho ${ }^{18}$. Essa assertiva vai ao encontro do que rege a Emenda Constitucional n. ${ }^{\circ}$ 64, de 2010, ${ }^{32}$ que alterou o texto do artigo $6^{\circ}$ da Constituição Federal e, por sua vez, instituiu o direito à alimentação como um direito social. Assim, a alimentação deve ser vista com um direito constitucional de todo cidadão, incluindo os indivíduos com necessidades especiais, que demanda uma alimentação especial.

A Segurança Alimentar e Nutricional do indivíduo vai além da disponibilidade diária de certa quantidade de alimento, ou seja, engloba também as condições higiênico-sanitárias e questões como a qualidade nutricional do alimento. Além disso, compreende também outros fatores, como 
a necessidade de atender às especificidades dos hábitos e práticas alimentares cultivadas ao longo da vida, de acordo com sua cultura e concepções religiosas, e de fornecer uma alimentação que seja adequada a sua saúde e melhore sua qualidade de vida.

\section{Considerações finais}

Vale ressaltar que não se pretende aqui exaurir o tema proposto, mas iniciar um debate que ainda se evidencia como sendo incipiente, sobretudo quando considera a concretude de uma violação de DHAA. Nesta perspectiva, afirma-se que os celíacos se enquadram como indivíduos com uma necessidade de alimentação especial e têm o direito ao acesso, à disponibilidade e à adequação de sua dieta, para que a mesma atenda a suas necessidades nutricionais e promova sua saúde e qualidade de vida.

\section{Referências}

1. Aaron L. The last two millennias echo-catastrophes are the driving forces for the potential genetic advantage mechanisms in celiac disease. Med hypotheses. 2011 Nov;77(5):773-6.

2. Silva TSG, Furlanetto TW. Diagnóstico de doença celíaca em adultos. Revista da Associação Médica Brasileira. 2010 fev;56(1):122-6.

3. Araújo HMC, Araújo WMC. Coeliac disease. Following the diet and eating habits of participating individuals in the Federal District, Brazil. Appetite. 2011 ago; 57(1):105-9.

4. Stevens L, Rashid M. Gluten-free and regular foods: a cost comparison. Can J Diet Pract Res. 2008 jan;69(3):147-50.

5. Peres AM et al. An electronic tongue for gliadins semi-quantitative detection in foodstuffs. Talanta. 2011 jan;83(3):857-64.

6. Kumar A et al. Latent celiac disease in reproductive performance of women. Fertil steril. 2011 mar;95(3):922-7.

7. Walker MM, Talley NJ. Clinical value of duodenal biopsies - Beyond the diagnosis of celiac disease. Pathol Res Pract. 2011 set;207(9):538-44.

8. Galvão LC et al. Apresentação clínica de doença celíaca em crianças durante dois períodos, em serviço universitário especializado. Arq Gastroenterol. 2004 dez;41(4):234-8.

9. Castro-Antunes MM et al. Celiac disease in first-degree relatives of patients. J pediatr (Rio J). 2010 ago;86(4):331-6.

10. Rubio-Tapia A et al. Increased prevalence and mortality in undiagnosed celiac disease. Gastroenterology. AGA Institute American Gastroenterological Association. 2009 jul;137(1):88-93. 
11. Strauch KA, Cotter VT. Celiac Disease: An Overview and Management for Primary Care Nurse Practitioners. J Nurse Pract. 2011 jul;7(7):588-99.

12. Kotze LMS. Celiac disease inBrazilian patients: associations, complications and causes of death. Forty years of clinical experience. Arq Gastroenterol. 2009 dez;46(4):261-9.

13. Ludvigsson JF et al. Increased suicide risk in celiac disease - a Swedish nationwide cohort study. Dig Liver Dis. 2011 ago;43(8):616-22.

14. Kotze LM da S. Doença celíaca. J Bras Gastroenterol. 2006;6(1):23-34.

15. Bai J, Zeballos E, Fried M, Corazza G, Schuppan D, Farthing M, et al. World Gastroenterology Organisation (WGO) Practice Guidelines: Celiac Disease. New York (US): World Gastroenterology Organisation; 2007.

16. Brasil. Ministério da Saúde. Política Nacional de Alimentação e Nutrição - PNAN. Brasília; 2012.

17. Lo Y-T et al. Dietary diversity and food expenditure as indicators of food security in older Taiwanese. Appetite. 2012 fev;58(1):180-7.

18. Pinheiro ARO, Carvalho MFCC. Transformando o problema da fome em questão alimentar e nutricional: uma crônica desigualdade social. Ciênc Saúde Coletiva. 2010 jan;15(1):121-30.

19. Freitas MCS, Pena PGL. Segurança Alimentar e nutricional: a produção do conhecimento com ênfase nos aspectos da cultura. Rev Nutr. 2007 fev;20(1):69-81.

20. Brasil. Presidência da República. Casa Civil. Lei no 11.346 de 15 de setembro de 2006. Cria o Sistema Nacional de Segurança Alimentar e Nutricional - SISAN com vistas em assegurar o direito humano à alimentação adequada e dá outras providências. Diário Oficial da União. Set 2006.

21. Burity $\mathrm{V}$ et al. Direito humano à alimentação adequada no contexto da segurança alimentar e nutricional. Brand; 2010.

22. Kepple AW, Segall-Corrêa AM. Conceituando e medindo segurança alimentar e nutricional. Ciênc Saúde Coletiva. 2011 jan;16(1):187-99.

23. Monteiro CA. Saúde no Brasil: contribuições para a agenda de prioridades de pesquisa. Brasília; 2004.

24. Poulain J-P, Proença RPC. Reflexões metodológicas para o estudo das práticas alimentares. Rev Nutr. 2003 dez;16(4):365-86.

25. Diez Garcia RW. Práticas e comportamento alimentar no meio urbano: um estudo no centro da cidade de São Paulo. Cad Saúde Pública. 1997 set;13(3):455-67.

26. Lee A, Newman JM. Celiac diet: its impact on quality of life. J Am Diet Assoc. 2003 nov;103(11):1533-5.

27. Araújo HMC et al. Doença celíaca, hábitos e práticas alimentares e qualidade de vida. Rev Nutr. 2010 jun; 23(3):467-74.

28. Singh J, Whelam K. Limited avaiability and higher cost of glúten-free foods. J Hum Nutr Diet. 2011 out;24(5):479-86. 
29. Brasil. Ministério do Desenvolvimento Social. Bolsa Família. Disponível em: http://www.mds.gov. $\mathrm{br} / \mathrm{search} / \mathrm{search}$ ?SearchableText=famílias $\% 20$ atendidas $\% 20 \&$ search_portal_type=File.

30. Pratesi R, Gandolfi L. Doença celíaca: a afecção com múltiplas faces. J Pediatr (Rio J). 2005;81(5):357-8.

31. Sdepanian VL, Morais MB, Fagundes-Neto U. Doença celíaca: características clínicas e métodos utilizados no diagnóstico de pacientes cadastrados na Associação dos Celíacos do Brasil. J Pediatr (Rio J). 2001 abr;77(2):232-9.

32. Brasil. Presidência da República. Casa Civil. Subchefia para Assuntos Jurídicos. Emenda Constitucional N. 64 de 04 de fevereiro de 2010. Altera o art. $6^{\circ}$ da Constituição Federal, para introduzir a alimentação como direito social. Diário Oficial da União 4 fev 2010. Disponível em: http://planalto.gov.br/ ccivil_03/Constituicao/Emendas/Emc?emc64.htm 
